\title{
Central venous catheter-related bloodstream infections (CVC-BSI) in patients of clinically suspected septicemia
}

\author{
Afroz $Z^{1}$, Jobayer $\mathrm{M}^{1}$, Ahmed $\mathrm{S}^{2}$, Anwar $\mathrm{S}^{2}$, Miah MRA ${ }^{2}$ \\ ${ }^{I}$ Department of Microbiology, Dhaka Medical College, Dhaka, Bangladesh, ${ }^{2}$ Department of \\ Microbiology and Immunology, Bangabandhu Sheikh Mujib Medical University, Dhaka, Bangladesh. \\ Email:zeenatafrozm35@gmail.com
}

\begin{abstract}
Central venous catheter-related bloodstream infections (CVC-BSI) are associated with morbidity and mortality especially in critically ill patients. This study was performed to find out the rate of CVC-BSI and CVC colonization, causative organism and their antibiogram in patients of Intensive Care Unit (ICU) and Department of Nephrology of tertiary care hospitals. A total of 100 patients from Bangabandhu Sheikh Mujib Medical University (BSMMU) and Dhaka Medical College Hospital (DMCH) who had CVC and clinically suspected of septicemia were included in the study. Paired CVC blood and peripheral venous blood (PVB) samples were collected from each patient and were cultured by automated blood culture method. CVC-BSI was diagnosed in $11 \%$ and CVC colonization in $43 \%$ patients by Differential time to positivity (DTP) method. Rate of CVC-BSI was 8/1000 CVC days and 11/1000 CVC days in BSMMU and DMCH respectively whereas CVC colonization rate was 32/1000 CVC days and 47.5/1000 CVC days in BSMMU and DMCH. The most common bacteria causing CVC-BSI was Klebsiella spp. (36.4\%) followed by Acinetobacter spp. (27.3\%), Pseudomonas spp. (18.2\%) and E. coli (18.2\%). Among bacteria isolated from CVC colonization majority were Pseudomonas spp. (30.23\%) and Staphylococcus epidermidis (30.23\%) followed by Acinetobacter spp. (27.91\%), Enterococcus spp. (6.98\%). Most of the isolated bacteria causing CVC-BSI were resistant to commonly used antibiotics, but showed good sensitivity to imipenem and colistin. Information about CVC-BSI, colonization and antibiogram of this study can help to guide the selection of suitable antibiotics for empirical therapy and to improve infection control measures of the hospital.
\end{abstract}

\section{Introduction}

Intravenous catheterization is one of the most common invasive procedures among patients admitted in hospitals. Vascular access poses significant potential risks of catheter related bloodstream infections. ${ }^{1}$ Central intravenous catheter are infected with the greatest frequency ${ }^{2}$ and central venous catheter-related bloodstream infection (CVC-BSI) is the third most frequent nosocomial infection and is a threat to patient's safety. ${ }^{3}$

There is a wide diversity between hospitals in the causative pathogens and their antimicrobial susceptibility pattern. Mansur et $\mathrm{al}^{4}$ in Bangladesh in a study reported that rate of CVCBSI is $14 \%$ and the most common causative pathogens were Pseudomonas, Acinetobacter and Staphylococcus aureus. Organisms causing CVC-BSI commonly are Pseudomonas spp, Acinetobacter spp, Klebsiella spp, Proteus spp, Enterobacter spp, E. coli, Staphylococcus aureus, coagulase negative Staphylococcus. ${ }^{5,6}$

Differential time to positivity (DTP) method is highly sensitive and specific for diagnosing CVC-BSI without removal of CVC. ${ }^{7,8}$ DTP has been defined as the time difference between the culture of CVC blood and peripheral venous blood (PVB) to become positive and the cutoff value of DTP is $\geq 120$ minutes. ${ }^{8}$ There are several denominators for detection of CVC-BSI rate, eg. CVC-BSI rate in $100 \mathrm{CVCs}$ or in $1000 \mathrm{CVC}$ days or in 100 patients. The most recommended denominator of detection of CVC-BSI rate is the 1000 CVC days. ${ }^{9}$ 
This study was designed to acquire information about the CVC-BSI rate, type of organism causing CVC-BSI and their antibiotic susceptibility pattern in tertiary care hospitals.

\section{Materials and Methods}

This cross sectional descriptive type of study was conducted during the period of July 2011 to June 2012.

Study population: Patients having clinical signs symptoms of septicemia who had CVC for more than 48 hours that was given after admission in Intensive Care Unit (ICU) and in Department of Nephrology.

Sample Size: A total of 100 patients (23 from ICU of BSMMU and 41 from ICU of DMCH and 36 patients from Department of Nephrology of $\mathrm{DMCH}$ ) were enrolled.

All laboratory works were performed in the Department of Microbiology and Immunology, BSMMU, Shahbagh, Dhaka.

Eight $\mathrm{ml}$ of blood was collected from both the CVC line and peripheral vein. After inoculation of blood into BACTAC culture vials they were transported to the Microbiology laboratory as soon as possible.

DTP was done by automated blood culture method as described by Raad et al. ${ }^{8}$ Paired vials (each vial containing $8 \mathrm{ml}$ of blood) were introduced into BACTEC 9240 machine (Dickinson and Company, Maryland, USA). All samples were incubated at $37^{\circ} \mathrm{C}$ up to culture positivity and the vials were discarded if no growth was detected within seven days.

The time difference between the CVC blood culture and PVB culture to become positive (DTP) was calculated by following way:

DTP of the blood sample (expressed in minutes) $=$ Time taken by peripheral venous blood culture to become positive - time taken by CVC blood culture to become positive

Positive sample of CVC blood and PVB were sub-cultured on blood agar and MacConkey agar and incubated aerobically at $37^{\circ} \mathrm{C}$ for 24 hours. If growth was present then identification of the organism was done by colony morphology, Gram staining and biochemical tests.

Fig. 1: Show the graphical presentation of detection of CVC-BSI by DTP method.

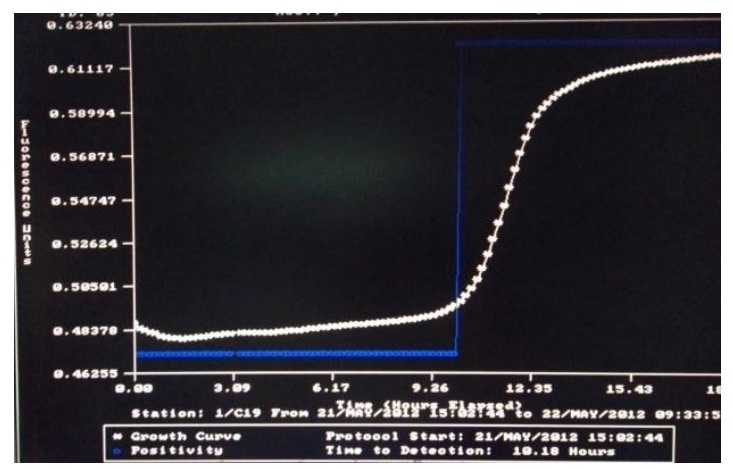

Figure - A

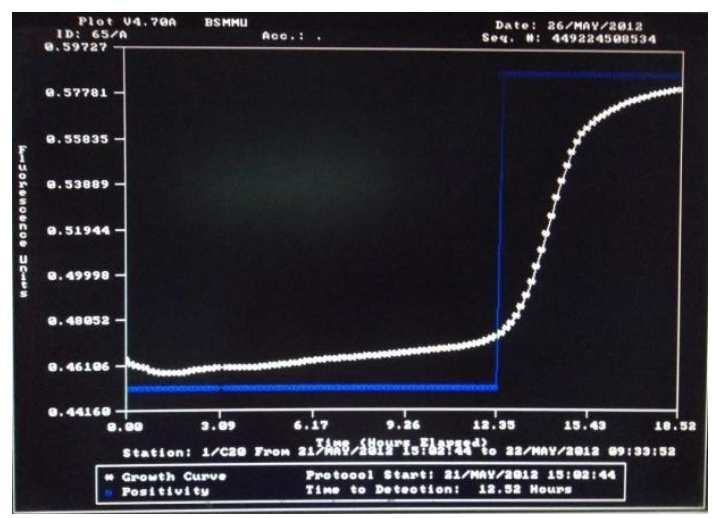

Figure - B

Fig. 1: Graphical presentation of detection of CVC-BSI by DTP method. Figure-A shows positive culture time (10.18 hrs.) of CVC blood and Figure-B shows positive culture time (12.52 hrs.) of PVB. The difference between CVC blood and PVB culture positivity is $>120$ minutes.

After organism identification antimicrobial sensitivity test was performed by disc diffusion method using Kirby-Bauer technique. ${ }^{10}$

1) Positive CVC-BSI: When difference in time between CVC blood culture positivity and PVB culture positivity is $\geq 120$ minutes and isolated organism is identical in genus, species and antibiotic susceptibility pattern.

2) Non CVC-BSI/ BSI source other than CVC:

a) When difference in time between CVC blood culture positivity and PVB culture positivity is 
$<120$ minutes or PVB culture become positive earlier than CVC blood culture and isolated organism is identical in genus, species and antibiotic susceptibility pattern.

b) If growth is present only in PVB culture but no growth in CVC blood culture.

3) CVC colonization: If growth is present only in CVC blood culture but no growth in PVB culture.

CVC-BSI rate was determined by the following method as mentioned by Salomao et al. ${ }^{11}$

CVC-BSI rate $=$ Total no. of CVC-BSI $/$ Total no. of CVC days X 1000

CVC day determination: Total number of days of exposure to CVC by all of the patients in selected population during the selected time period.

Ethical consideration: The protocol of this study was approved by Ethical Clearance Committee (ERC) of Bangabandhu Sheikh Mujib Medical University. Informed written consent was taken from the patient or from their attendant before collection of blood samples. The anonymity of the participants and confidentiality of information was maintained strictly.

\section{Result}

Out of 100 patients, CVC-BSI was diagnosed in $11 \%$, CVC colonization in $43 \%$, Non-CVC-BSI in $10 \%$ patients. Among 23 patients of ICU of BSMMU, CVC-BSI was detected in $8.70 \%$, CVC colonization in $34.78 \%$ and Non-CVC-BSI in $17.40 \%$ patients, whereas in 41 patients of ICU of DMCH, CVC-BSI was detected in $14.63 \%$ and CVC colonization in $43.90 \%$ and Non-CVC-BSI in $7.32 \%$ patients. Out of 36 patients, CVC-BSI was detected in $8.33 \%$ and CVC colonization in $47.22 \%$, and Non-CVC-BSI in $8.33 \%$ patients of department of Nephrology of DMCH (Table I).

Table II shows the rate of CVC-BSI and CVC colonization in $1000 \mathrm{CVC}$ days in two institutes. Rate of CVC-BSI was 8/1000 CVC days in BSMMU and $11 / 1000$ CVC days in DMCH whereas CVC colonization rate was $32 / 1000$
CVC days in BSMMU and 47.5/1000 CVC days in $\mathrm{DMCH}$.

Table I: Rate of CVC-BSI and CVC colonization detected among the patients of different institutes

\begin{tabular}{ccccc}
\hline Institutes & Patients & $\begin{array}{c}\text { CVC- } \\
\text { BSI } \\
\mathrm{n}(\%)\end{array}$ & $\begin{array}{c}\text { CVC } \\
\text { colonization } \\
\mathrm{n}(\%)\end{array}$ & $\begin{array}{c}\text { Non CVC-BSI } \\
\mathrm{n}(\%)\end{array}$ \\
\hline $\begin{array}{c}\text { BSMMU } \\
\text { ICU }\end{array}$ & 23 & $2(8.7)$ & $8(34.8)$ & $4(17.4)$ \\
DMCH & & & & \\
ICU & 41 & $6(14.6)$ & $18(43.9)$ & $3(7.3)$ \\
$\begin{array}{c}\text { Dept. of } \\
\text { Nephrology } \\
\text { Total }\end{array}$ & 36 & $3(8.3)$ & $17(47.2)$ & $3(8.3)$ \\
\hline
\end{tabular}

CVC-BSI: Central venous catheter related bloodstream infection CVC colonization: Central venous catheter colonization

Table II: Rate of CVC-BSI and CVC colonization in $1000 \mathrm{CVC}$ days among the episodes.

\begin{tabular}{|c|c|c|c|c|}
\hline \multirow[b]{2}{*}{$\begin{array}{l}\text { Type of } \\
\text { infection }\end{array}$} & \multicolumn{2}{|c|}{ BSMMU } & \multicolumn{2}{|c|}{ DMCH } \\
\hline & $\begin{array}{l}\frac{\pi}{0} \cdot \overrightarrow{1} \\
\frac{0}{0} \\
\stackrel{0}{0} \\
\frac{0}{2}\end{array}$ & $\begin{array}{c}\text { Episodes } \\
/ 1000 \\
\text { CVC } \\
\text { days }\end{array}$ & $\begin{array}{l}\frac{\pi}{0} \cdot \overrightarrow{0} \\
\stackrel{0}{0} \\
\stackrel{D}{0}\end{array}$ & $\begin{array}{c}\text { Episodes/1 } \\
000 \text { CVC } \\
\text { days }\end{array}$ \\
\hline CVC-BSI & 2 & 8 & 9 & 11 \\
\hline $\begin{array}{c}\text { CVC } \\
\text { Colonization }\end{array}$ & 8 & 32 & 35 & 47.5 \\
\hline
\end{tabular}

CVC days: Central venous catheter days.

Bacteria causing CVC-BSI and CVC colonization were isolated from 54 patients. Among 11 cases of CVC-BSI, the isolated organisms were Klebsiella spp. $36.4 \%$, Acinetobacter spp. $27.3 \%$, Pseudomonas spp. $18.2 \%$ and E. coli $18.2 \%$. Among 43 bacteria isolated from CVC colonization Pseudomonas spp. were $30.23 \%$, Staphylococcus epidermidis $30.23 \%$, Acinetobacter spp. 27.91\%, Enterococcus spp. 6.98\%, Klebsiella spp and Staphylococcus aureus was $2.33 \%$ (Table III).

Among the isolated Acinetobacter spp. from CVC-BSI patients, $66.67 \%$ were sensitive to colistin and imipenem, $33.33 \%$ was sensitive to cotrimoxazole. 
Table III: Bacteria isolated from CVC-BSI and CVCcolonization.

\begin{tabular}{ccc}
\hline Isolated organism & $\begin{array}{c}\text { CVC-BSI } \\
(\mathrm{n}=11)\end{array}$ & $\begin{array}{c}\text { CVC } \\
\text { Colonization } \\
(\mathrm{n}=43)\end{array}$ \\
\hline $\begin{array}{c}\text { Acinetobacter spp. } \\
(\mathrm{n}=15)\end{array}$ & $3(27.3 \%)$ & $12(27.91 \%)$ \\
$\begin{array}{c}\text { Pseudomonas spp. } \\
(\mathrm{n}=15)\end{array}$ & $2(18.2 \%)$ & $13(30.23 \%)$ \\
$\begin{array}{c}\text { Klebsiella spp. } \\
(\mathrm{n}=5)\end{array}$ & $4(36.4 \%)$ & $1(2.33 \%)$ \\
$\begin{array}{c}\text { E. coli } \\
(\mathrm{n}=2)\end{array}$ & $2(18.2 \%)$ & $0(0 \%)$ \\
$\begin{array}{c}\text { Staph. epidermidis } \\
(\mathrm{n}=13)\end{array}$ & $\mathrm{Nil}$ & $13(30.23 \%)$ \\
$\begin{array}{c}\text { Enterococcus spp. } \\
(\mathrm{n}=3)\end{array}$ & $\mathrm{Nil}$ & $3(6.98 \%)$ \\
$\begin{array}{c}\text { Staph. aureus } \\
(\mathrm{n}=1)\end{array}$ & $\mathrm{Nil}$ & $1(2.33 \%)$ \\
\hline
\end{tabular}

All Pseudomonas spp. were sensitive to imipenem, $50 \%$ was sensitive to ceftazidime and colistin; $100 \%$ E. coli were sensitive to gentamicin, $50 \%$ was sensitive to cotrimoxazole and imipenem. All Klebsiella spp. were sensitive to gentamicin and imipenem, $50 \%$ were sensitive to cotrimoxazole and $25 \%$ was sensitive to amikacin (Table IV).

Table IV: Antibiotic susceptibility pattern of bacteria isolated from CVC-BSI.

\begin{tabular}{cccccc}
\hline Bacteria & \multicolumn{5}{c}{ Sensitivity (\%) } \\
\hline & Cip & Cot & G & Cro & Caz \\
\hline Klebsiella & 0 & 50 & 100 & 0 & 0 \\
Acinetobacter & 0 & 33.3 & 0 & 0 & 0 \\
E. coli & 0 & 50 & 100 & 0 & 0 \\
Pseudomonas & 0 & - & 0 & - & 50 \\
\hline & Ak & Imp & Net & TZP & CT \\
\hline Klebsiella & 25 & 100 & 0 & - & - \\
Acinetobacter & 0 & 66.7 & 0 & 0 & 66.7 \\
E. coli & 0 & 50 & 0 & - & - \\
Pseudomonas & 0 & 100 & 0 & 0 & 50 \\
\hline
\end{tabular}

Cip-ciprofloxacin, Cot-Co-trimoxazole, G-gentamicin, Croceftriaxone, Caz-ceftazidime, Ak-amikacin, Imp-imipenem, Netnetilmicin, TZP-piperacillin/tazobactum, CT-colistin.

\section{Discussion}

Central venous catheter-related bloodstream infections (CVC-BSI) are common type of nosocomial bloodstream infections. ${ }^{6,12}$ Although there are valid guidelines for good practice in central venous catheterization, CVC-BSI still is a serious problem especially in patients with long term catheterization. ${ }^{13}$

CVC-BSI and CVC colonization were detected by DTP method which is a newer and valid method for CVC-BSI diagnosis without removing the CVC. CVC-BSI rate was detected in $11 \%$ patients, CVC colonization $43 \%$ and non CVC-BSI was $10 \%$ in this study by DTP method. A cutoff value of DTP $\geq 120$ minutes was taken as standard value for diagnosing CVC-BSI which is supported by the findings of Blot et al. ${ }^{14}$ who found that DTP of $\geq 120$ minutes was sensitive and specific for diagnosing CVC-BSI.

The rate of CVC-BSI and CVC colonization were lower in patients of ICU of BSMMU than the patients of ICU of DMCH. The reason of low CVC-BSI in patients of ICU of BSMMU may be due to better infection control practice, such as CVC care, nurse patient ratio, visitor restriction, waste disposal etc. which is supervised by an infection control team. Holton et al. ${ }^{9}$ mentioned that effective infection control system, standardized CVC care and patient's immune status may contribute to the lower rate of CVCBSI.

CVC colonization was found in $43 \%$ cases. Ideally intravenous catheters should remain sterile because bacteria from the colonization may cause silent infection and eventually clinical sepsis if the colonized catheters remain in situ for longer period. ${ }^{5}$ Such kind of colonized catheter should not be disregarded and as these organisms are potential pathogens that may sometimes cause diseases in the host these colonizing bacteria should be considered as a threat and patients with colonized catheters should be followed up for features of bloodstream infection.

The rate of CVC-BSI was found 8/1000 CVC days and 11/1000 CVC days in BSMMU and $\mathrm{DMCH}$ respectively whereas CVC colonization rate was 32/1000 CVC days and 47.5/1000 CVC 
days in BSMMU and in DMCH. In a similar study at BIRDEM, Dhaka4 CVC-BSI rate was found 16/1000 CVC days and CVC colonization was 48.3/1000 CVC days. Such higher CVC-BSI rate was also reported in several studies in India, ${ }^{15}$ Taiwan ${ }^{16}$ and in Brazil ${ }^{17}$ ranging from 7.5 to $10.2 / 1000 \mathrm{CVC}$ days. The rate of CVCBSI was 1.5 to $2.9 / 1000 \mathrm{CVC}$ days in different ICUs of CDC National Healthcare Safety Network (NHSN) hospitals in the United States ${ }^{18}$ which is lower than this study. Developing countries have insufficient infection control measures, low nurse-to-patient ratio in hospital, limited financial or administrative support which may be responsible for higher CVC-BSI ${ }^{17}$. CVC colonization was also high in this study which might be due to prolong duration of CVC placement. Cronin et $\mathrm{al}^{19}$ reported $27 \%$ colonization in his study and mentioned that improper manipulation and prolonged placement of $\mathrm{CVC}$ is the reason for frequent $\mathrm{CVC}$ colonization.

Higher number of Gram negative rods was isolated from CVC-BSI and CVC colonization in this study. Highest rate of isolated bacteria was Klebsiella spp. followed by Acinetobacter spp., Pseudomonas spp., and E. coli. This finding correlated with the study by Bicudo et al. ${ }^{17}$ in Brazil who showed prevalent organism of CVCBSI was Acinetobacter spp. followed by Klebsiella spp. and Pseudomonas spp. Mansur et al. ${ }^{4}$ in Bangladesh found Pseudomonas and Acinetobacter were the major causative agents of CVC-BSI that is also in accordance with the present study. Higher rate of Gram negative rods was also reported in several other studies. ${ }^{20-22}$

In this study, Staphylococcus was not found as causative agent of CVC-BSI but coagulase negative Staphylococcus and Staph aureus caused CVC colonization in $30.23 \%$ and $2.33 \%$ cases respectively. Mansur et al. ${ }^{4}$ observed only CVC colonization by coagulase negative Staphylococcus and Staph aureus but no CVCBSI caused by them.

Most of the organisms causing CVC-BSI were resistant to common antibiotics used in ICU of BSMMU and DMCH. All isolated Gram negative bacilli were resistant to ciprofloxacin, ceftriaxone and netilmicin. Klebsiella spp. and E. coli were also $100 \%$ resistant to ceftazidime. Pseudomonas spp. showed $100 \%$ resistance to tazobactam-piperacillin, gentamicin and amikacin; Acinetobacter spp. were $100 \%$ resistant to gentamicin, amikacin, ceftazidime and tazobactam-piperacillin. This sensitivity pattern was consistent with the antibiogram of organism isolated from ICU patients of CVCBSI in several studies done in Brazil ${ }^{11}$ and India. ${ }^{15}$

Possible explanation of infection by these resistant strains in ICU is the selection pressure exerted by extensive use of antibiotics and may be due to transmission of resistant clones between the patients. ${ }^{23}$ Infections with resistant strains are transmitted among the patients due to inadequate implementation of infection control measures in institutes. ${ }^{24}$

Conclusion: The rate of CVC-BSI and CVC colonization were significant and the isolated organisms from CVC-BSI were resistant to most of the common antibiotics used. Knowledge of this antimicrobial susceptibility pattern may aid to make an empirical antibiotic protocol.

\section{Acknowledgement:}

We acknowledge the department of ICU of BSMMU \& DMCH and Department of Nephrology for providing support for the study.

\section{References}

1. Rosenthal VD. Central line-associated bloodstream infections in limited-resource countries: a review of the literature. Clin Infect Dis 2009; 49(12):1899907.

2. Raad I and Darouiche R. Prevention of infections associated with intravascular devices. Curr Opin Crit Care 1996; 2: 361-62.

3. Richards MJ, Edwards JR, Culver DH, Gaynes RP. Nosocomial infections in combined medical-surgical intensive units in the United States. Infect Control Hosp Epidemiol 2000; 21: 510-15.

4. Mansur FJ, Barai L, Karim MM, Haq JA, Fatema K, Faruq MO. Intravascular catheter related infection and antimicrobial susceptibility pattern of isolated bacteria in a tertiary care hospital of Bangladesh. Indian J Med Microbial 2014; 32(1): 68-71. 
5. Brun-Buisson $C$, Abrouk F, Legrand P, Huet $Y$, Larabi S, Rapin M. Diagnosis of Central venous catheter-related sepsis-critical level of quantitative tip culture. Arch Intern Med 1987; 147(5): 873-77.

6. Maki DG and Mermel LA. Infections due to infusion therapy. In: Bennett JV and Brachman PS. Eds. Hospital infections, 4th ed. Philadelphia: Lippincott-Raven, 1998: 689-724.

7. Blot F, Schimidt E, Nitenberg G, Tancrede C, Leclercq B, Laplanche A, et al. Earlier positivity of central-venous- versus peripheral-blood cultures is highly predictive of catheter-related sepsis. J Clin Microbiol 1998; 36(1): 105-09.

8. Raad I, Hanna HA, Alakech B, Chatzinikolaou I, Johnson MM, Tarrand J. Differential time to positivity: a useful method for diagnosing catheterrelated bloodstream infections. Ann Intern Med 2004; 140(1): 18-25.

9. Holton D, Panton S, Conly J, Embree J, Taylor G, Thompson W. Central venous catheter-associated bloodstream infections occurring in Canadian intensive care units: A six-month cohort study. Can J Infect Dis Med Microbiol 2006; 17(3): 169-76.

10. Bauer AW, Kirby WMM, Sherris JC, Turck M. Antibiotic susceptibility testing by a standardized single disc method. Am J Clin Pathol 1966; 36: 49396.

11. Salomao R, Rosenthal VD, Grimberg G, Nouer S, Blecher S, Buchner-Ferreira $\mathrm{S}$, et al. Deviceassociated infection rates in intensive care units of Brazilian hospitals: findings of the International Nosocomial Infection Control Consortium. Pan Am J Public Health 2008; 24(3): 195-202.

12. Jarvis WR, Edwards JR, Culver DH, Hughes JM, Horan T, Emori TG, et al. Nosocomial infection rates in adult and pediatric intensive care units in the United States. National Nosocomial Infections Surveillance System. Am J Med 1991; 91: 185-91.

13. Astagneau P, Maugat S, Tran-Minh T, Douard M, Longuet $\mathrm{P}$, Maslo C, et al. Long-term central venous catheter infection in HIV-infected and cancer patients: a multicenter cohort study. Infect Control Hosp Epidemiol 1999; 20: 494-98.

14. Blot F, Nitenberg G, Chachaty E, Raynard B, Germann N, Antoun S, et al. Diagnosis of catheterrelated bacteraemia: a prospective comparison of the time to positivity of hub-blood versus peripheralblood cultures. Lancet 1999; 354: 1071-77.

15. Mehta A, Rosenthal VD, Mehta Y, Chakravarthy M, Todi SK, Sen N, et al. Device-associated nosocomial infection rates in intensive care units of seven Indian cities. Findings of the International Nosocomial Infection Control Consortium (INICC). J Hosp Infect 2007; 67: 168-74.

16. Chen H, Wang F, Lin M, Lin Y, Huang L, Liu C. Risk factors for central venous catheter-related infections in general surgery. J Microbiol Immunol Infect 2006; 39: 231-36.

17. Bicudo D, Batista R, Furtado GH, Sola A, deMedeiros EAS. Risk factors for catheter-related bloodstream infection: a prospective multicenter study in Brazilian intensive care units. Braz J Infect Dis 2011 ; 15(4): 328-31.

18. Edwards JR, Peterson KD, Andrus ML, Tolson JS, Goulding JS, Dudeck MA, et al. National Healthcare Safety Network (NHSN) Report, data summary for 2006, issued June 2007. Am J Infect Control 2007; 35: 290-301.

19. Cronin WA, Germanson TP, Donowitz LG. Intravascular catheter colonization and related bloodstream infection in critically ill neonates. Infect Control Hosp Epidemiol 1990; 11: 301-08.

20. Tan CC, Zanariah Y, Lim KI, Balan S. Central venous catheter related bloodstream infection: Incidence and an analysis of risk factors. Med $\mathbf{J}$ Malaysia 2007; 62(5): 370-74.

21. Chatzinikolaou L and Raad I. Catheter related infections. Iranian J Clin Infect Dis 2006; 1(2): 7987.

22. Kurtz P, Rosa P, Penna G, Braga F, Kezen J, Drumond LE, et al. Antibiotic coated catheter to decrease infection. Pilot study. Rev Bras Ter Intensiva 2008; 20(2): 160-64.

23. Meyer E, Jonas D, Schwab F, Reuden H, Gastmeier $\mathrm{P}$, Daschner FD. Design of a surveillance system of antibiotic use and bacterial resistance in German intensive care units (SARI). Infect 2003; 31(4): 20815.

24. Rello J and Diaz E. Acinetobacter baumannii: a threat for the ICU? Intensive Care Med 2003; 29 : $350-51$. 